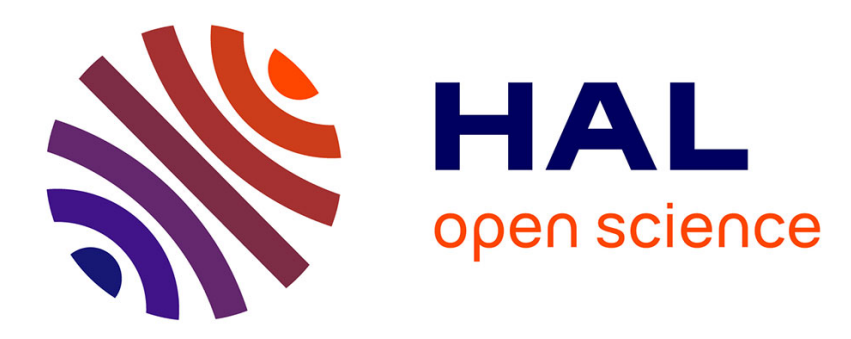

\title{
Sur l'approche matricielle des rapports de propriété et la théorie du contrôle
}

Philippe Bance

\section{To cite this version:}

Philippe Bance. Sur l'approche matricielle des rapports de propriété et la théorie du contrôle. Revue Economique, 1988, 39 (2), pp.421-440. 10.2307/3501910 . hal-01965200

HAL Id: hal-01965200

https://hal-normandie-univ.archives-ouvertes.fr/hal-01965200

Submitted on 25 Dec 2018

HAL is a multi-disciplinary open access archive for the deposit and dissemination of scientific research documents, whether they are published or not. The documents may come from teaching and research institutions in France or abroad, or from public or private research centers.
L'archive ouverte pluridisciplinaire HAL, est destinée au dépôt et à la diffusion de documents scientifiques de niveau recherche, publiés ou non, émanant des établissements d'enseignement et de recherche français ou étrangers, des laboratoires publics ou privés. 


\section{Sur l'approche matricielle des rapports de propriété et la théorie} du contrôle

Monsieur Philippe Bance

\section{Citer ce document / Cite this document :}

Bance Philippe. Sur l'approche matricielle des rapports de propriété et la théorie du contrôle. In: Revue économique, volume $39, n^{\circ} 2,1988$. pp. 421-440;

doi : $10.2307 / 3501910$

https://www.persee.fr/doc/reco_0035-2764_1988_num_39_2_409071

Fichier pdf généré le 28/03/2018 


\begin{abstract}
On the matrix approach of property relations and the control theory

Legal and economic property relations (or possession and control relations) are not exacdy of the same kind. This duality can be apprehended by the matrix approach which enable to build gap indicators of property relations. However, this gap must not be understood in a narrow sense by assimilating it to a difference between possession and control only associated with differential rates of contribution in economic units. The reason is that control gaps proceed from extra-patrimonial domination or also from public behavioural specificities. This article deals with these control gaps and the way they contribute to the whole gap in property relations.
\end{abstract}

\title{
Résumé
}

Sur l'approche matricielle des rapports de propriété et la théorie du contrôle

Les rapports de propriété juridique (ou de possession) et de propriété économique (ou de contrôle) ne sont parfaitement identifiables. Cette dualité peut être appréhendée par l'approche matricielle qui permet de construire des indicateurs de décalage des rapports de propriété. Ce décalage ne doit cependant pas être perçu de manière restrictive en l'assimilant à un écart possession-contrôle associé uniquement à des différentiels de taux de participation dans les unités économiques. Car des décalages de contrôle naissent de dominations extra-patrimoniales ou encore de spécificités comportementales publiques. Ce sont ces décalages de contrôle et leur contribution au décalage total des rapports de propriété qui sont appréhendés dans cet article. 


\title{
Sur l'approche matricielle des rapports de propriété et la théorie du contrôle
}

Philippe Bance *

\begin{abstract}
Les rapports de propriété juridique (ou de possession) et de propriété économique (ou de contróle) ne sont parfaitement identifiables. Cette dualité peut être appréhendée par l'approche matricielle qui permet de construire des indicateurs de décalage des rapports de propriété. Ce décalage ne doit cependant pas être perçu de manière restrictive en l'assimilant à un écart possession-contróle associé uniquement à des différentiels de taux de participation dans les unités économiques. Car des décalages de contróle naissent de dominations extra-patrimoniales ou encore de spécificités comportementales publiques. Ce sont ces décalages de contrôle et leur contribution au décalage total des rapports de propriété qui sont appréhendés dans cet article.
\end{abstract}

Les systèmes productifs occidentaux ont connu depuis plusieurs décennies de très profondes mutations : la prolifération des liens financiers en a considérablement modifié la configuration sur fonds de concentration, de restructurations, d'internationalisation. Les ramifications croissantes, l'imbrication et l'interpénétration toujours plus poussées des patrimoines mobiliers des agents économiques - que l'on peut taxer de fluidification des rapports de propriété juridique - rendent en fait singulièrement difficile l'appréhension des rapports de propriété économique (c'est-à-dire de contrôle) prévalant au sein de ces systèmes productifs.

En effet, les rapports de propriété économique ne sont pas réductibles aux rapports de propriété juridique bien qu'ils y trouvent leur fondement principal ${ }^{1}$. Et la complexification des structures juridique est source

* Nous remercions Lionel Monnier qui fut à l'origine de cet article ainsi que les deux rapporteurs anonymes de la Revue économique et André Lapidus pour avoir contribué, grâce à leurs commentaires constructifs, à améliorer la version initiale de ce papier.

1. La question de la séparation entre la propriété et le contrôle a retenu l'attention des économistes depuis la fin du xix siècle. Les controverses, pendant les années 1930, auxquelles donna lieu la publication des travaux de A. Berle et G. Means (The modern corporation and private property, 19.32), constituent une excellente illustration de cette opposition, aujourd'hui encore irréductible, entre les thèses qui voient surtout dans la propriété une source de pouvoir matériel et celles qui, préfigurant les conceptions développées dans la théorie moderne des droits de propriété, en font avant tout le moyen d'assurer la prééminence des rapports d'échange. Cf. J. Magnan de Bornier, " Propriété éclatée ou propriété 
d'un décalage grandissant entre rapports de possession et rapports de contrôle. Dans ces conditions, construire un outil mathématique qui rende compte des rapports de propriété existant au sein du système productif est ouvre doublement utile : elle permet non seulement d'appréhender, de clarifier les rapports de possession et de contrôle mais également de mesurer le décalage qui existe entre eux. C'est dans cet esprit que, dans son ouvrage Théorie économique du patrimoine, F. Morin 1 propose une formalisation matricielle des rapports de propriéte. Nous en rappellerons les grandes lignes. Cependant, cette thèse, bien que parfaitement valide dans ses fondements, peut à notre sens être améliorée par une perception plus élaborée de notion de contrôle. Aussi procéderonsnous à une reformulation plus conforme à la réalité de l'appropriation économique et par là même au décalage effectif des rapports de propriété.

\section{LES FONDEMENTS D'UNE THÉORIE DU CONTROLE}

La dissociation des agents économiques constitue le préalable d'une formalisation des structures juridiques qui permet, dans l'analyse de F. Morin, de déterminer les structures de pouvoir prévalant au sein des économies capitalistes.

\section{La dissociation des agents}

Deux types d'agents sont identifiables : ceux contre lesquels aucun titre de propriété ne peut être émis (appelés unités économiques finales) et ceux, dénommés unités économiques intermédiaires, qui sont détenus en toute propriété par d'autres agents (finaux ou non).

Dans la première catégorie figurent ainsi les personnes physiques (ou plus largement les ménages), les collectivités publiques et en particulier l'Etat qui apparaissent comme les véritables possesseurs (par voie directe ou indirecte) du patrimoine national. Quant à la catégorie des agents intermédiaires, elle rassemble les entreprises de toutes natures, y compris les entreprises individuelles (que l'on distingue du patrimoine des entrepreneurs individuels).

dispersée n, contribution à la Journée d'Etude : . La propriété en économie théorique ", Amiens, 30 mai 1986. Voir également le numéro spécial de la Revue économique, \& La propriété ", 38 (6), novembre 1987. 1984.

1. Cf. F. Morin, Théorie économique du patrimoine, Ellipses, Editions marketing, 


\section{La formalisation des structures juridiques}

\section{La construction de matrices de possession immédiate}

Les rapports de possession immédiate peuvent être appréhendés simplement, en construisant deux matrices (une par type d'agents) où figurent les taux de participation de chaque agent dans les unités économiques intermédiaires.

Fixons à $q$ et à $p$ les nombres respectifs d'agents intermédiaires et finaux. La matrice des agents intermédiaires $\left(\mathbf{M}_{\mathbf{I}}^{\mathrm{PI}}\right)$ est donc une matrice carrée d'ordre $q$ à coefficients rationnels compris entre 0 et 1 :

$$
M_{1}^{\mathrm{PI}}=\left(\begin{array}{ll}
\alpha & i
\end{array}\right) \quad(i=1,2, \ldots, q), \quad(j=1,2, \ldots, q)
$$

avec $\mathbf{M}_{\mathbf{r}}^{\mathrm{Px}} \in \mathbf{M}_{q} q(\mathrm{~V})$ où $\mathbf{V}=\{x \in \mathrm{Q} / x \in[0,1]\}$

et où $\alpha i j$ est le taux de participation de l'unité intermédiaire $i$ dans l'unité intermédiaire $j$.

Des remarques similaires peuvent être émises quant à la matrice des détenteurs finaux à cette différence près qu'il s'agit d'une matrice rectangulaire à $p$ lignes et $q$ colonnes.

$$
\mathbf{M}_{\mathrm{P}}^{\mathrm{PX}}=\left(\beta h_{i}\right) \quad(h=1,2, \ldots, p), \quad(j=1,2, \ldots, q)
$$

avec $\mathbf{M}_{\mathbf{P}}^{\mathbf{P I}} \in \mathbf{M} p, q(\mathrm{~V})$.

Par construction et du fait de l'exhaustivité supposée du recensement des participations, la somme des taux de participation des agents intermédiaires et finaux dans chaque unité intermédiaire est égale à $100 \%$. Donc :

$$
\alpha \cdot j+\beta \cdot j=1 \quad(\forall i=1,2, \ldots, q)
$$

ou encore $\mathrm{U} q \cdot \mathbf{M}_{\mathrm{I}}^{\mathrm{PI}}+\mathrm{U} p \cdot \mathbf{M}_{\mathrm{F}}^{\mathrm{PI}}=\mathbf{U} q$.

$U p$ et $U q$ étant respectivement les vecteurs lignes de dimension $(1, p)$ et $(1, q)$ à coefficients tous égaux à l'unité (pour simplification, nous transcrirons indifféremment par $U$ les vecteurs $U q$ et $U p$ ).

\section{La détermination des matrices de possession ultime}

De la relation précédente, on tire :

$$
\mathrm{U} p \cdot \mathrm{M}_{\mathrm{P}}^{\mathrm{PI}}=\mathrm{U} q \cdot\left(\mathrm{I} q-\mathrm{M}_{\mathrm{I}}^{\mathrm{PI}}\right) \text { où } \mathrm{I} q \text { est la matrice unité d'ordre } q
$$

(nous l'écrirons simplement I). 
Si $\left(\mathbf{I}-\mathbf{M}_{\mathbf{I}}^{\mathrm{PI}}\right)$ est non singulière ${ }^{1}$, cela équivaut à :

$$
\mathbf{U} \cdot \mathbf{M}_{\mathbf{P}}^{\mathrm{PI}} \cdot\left(\mathbf{I}-\mathbf{M}_{\mathbf{I}}^{\mathbf{P I}}\right)^{-1}=\mathbf{U}
$$

Posons $M_{F}^{\text {PU }}=M_{F}^{P I} \cdot\left(I-M_{I}^{P I}\right)^{-1} \cdot$ Par construction $M_{F}^{P \mathbb{V}} \in M p, q(V)$. $M_{F}^{P V}=(\gamma h j)$ est en fait la matrice de possession ultime des agents finaux où $\gamma h j$ représente la participation cumulative (de quelque rang que ce soit) de l'agent final $h$ dans l'unité intermédiaire $j^{2}$. Par ailleurs, $\gamma \cdot j=1(\forall j=1,2, \ldots, q)$ conformément à l'hypothèse de possession ultime totale de chaque unité intermédiaire par les agents finaux.

De manière similaire, on peut construire la matrice de possession ultime des unités intermédiaires :

$$
\begin{gathered}
M_{I}^{P U}=M_{I}^{P I} \cdot\left(I-M_{I}^{P I}\right)^{-1} \\
\left.M_{I}^{P U}=(\theta i j) \in M_{q}(V) \text { avec } 0 \leqslant \theta \cdot i \leqslant 1 \quad \forall \forall i=1,2, \ldots, q\right)^{2}
\end{gathered}
$$

\section{La formalisation des rapports de propriété économique}

Les rapports de propriété économique se différencient des rapports d'appropriation juridique, car ils renvoient à la notion de contrôle et non plus à la détention de titres de propriété. Il va donc s'agir de déterminer matriciellement les « chaînes de contrôle » qui existent au sein de l'ensemble des unités économiques intermédiaires et d'identifier les agents de contrôle ultime.

1. La non-singularité de $\left(\mathrm{I}-\mathrm{M}_{\mathrm{I}}^{\mathrm{PI}}\right)$ n'est pas garantie, contrairement à ce qui est indiqué dans l'ouvrage de $F$. Morin. Comme $M_{\mathrm{I}}^{\mathrm{PI}}$ est une matrice carrée non négative, on sait d'après les théorèmes de Frobénius (voir, par exemple, A. Takayama, Mathematical economics, $2^{2}$ édit., Cambridge, Cambridge University Press, p. 367 394) que $\left(\varphi \cdot I-M_{I}^{\text {PI }}\right)$, où $\varphi$ est un réel positif, est régulière et possède une matrice inverse semi-positive si, et seulement si, $\lambda$, racine de Frobénius de $M_{I}^{P 1}$, est strictement inférieur à $\varphi$. Par ailleurs, si l'on appelle $s$ la plus petite des sommes de chacune des colonnes de $M_{\mathbf{I}}^{\mathrm{PI}}$ et $S$, la plus grande, est vérifiée la relation suivante $: s \leqslant \lambda \leqslant S$. Par définition de $\mathrm{M}_{\mathrm{I}}^{\mathrm{PI}}$, on a donc $0 \leqslant \lambda \leqslant 1$. Deux cas de figure sont alors à envisager :

- si $M_{I}^{\text {PI }}$ est indécomposable, 'l'égalité entre $\lambda$ et $S$ est vérifiée si, et seulement si, $s=\mathrm{S}$. Or ceci est impossible car la matrice (semi-positive) des agents finaux complète $M_{I}^{P I}$ de telle sorte que lon obtienne des sommes de colonne égales à l'unité. Comme $\lambda<1$, $\left(I-M_{I}^{\mathrm{PI}}\right)$ est donc régulière et a une matrice inverse semi-positive ;

- si $\mathbf{M}_{\mathbf{I}}^{\mathrm{PI}}$ est décomposable, on ne peut définitivement conclure :

- pour $\lambda \neq 1,\left(I-M_{I}^{\text {PI }}\right)$ est régulière et possède une matrice inverse semipositive,

- pour $\lambda=1$, (I - $\left.M_{I}^{P I}\right)$ est, en revanche, singulière; un cas de figure de ce type peut survenir en présence d'un sous-ensemble de participations circulaires pour lesquelles la possession ultime n'existerait pas.

2. Voir F. Morin, Théorie économique du patrimoine, op. cit., p. 15-16. N.B. : le raisonnement ne vaut cependant que si la racine de Frobénius de $M_{I}^{P I}$ diffère de l'unité, condition de régularité de $\left(I-M_{\mathbf{I}}^{\mathbf{P I}}\right)$. 


\section{La construction des matrices de contrôle immédiat}

Les matrices de possession immédiate servent de support à la construction des matrices de contrôle immédiat : leur lecture permet d'appréhender, compte tenu de pratiques juridiques connexes (vote bloqué, vote plural), les détenteurs du pouvoir de contrôle immédiat au sein de chaque unité économique intermédiaire. Les coefficients de la matrice de contrôle immédiat des agents intermédiaires (que nous noterons $\mathbf{M}_{\mathrm{I}}^{\mathrm{GI}}$ avec $\left.\mathbf{M}_{\mathbf{I}}^{\mathrm{CI}}=(\lambda i j) \in \mathbf{M}_{q}(\mathrm{~V})\right)$ s'établissent comme suit :

- si la participation au capital de l'unité intermédiaire $i$ dans l'unité $j$ lui en assure le contrôle, alors $\lambda i j=1 / n, n$ étant le nombre d'unités contrôlant $j(\lambda i j=1$ si $i$ est la seule unité qui contrôle $j)$;

- si la participation de $i$ dans $j$ ne suffit pas au contrôle, alors $\lambda i j=0$.

Cette méthode vaut bien entendu pour la matrice de contrôle immédiat des agents finaux : $\mathbf{M}_{P}^{\mathrm{I}}=(\varphi h j) \in \mathbf{M} p, q(\mathrm{~V})$.

Le ou les agent(s) de contrôle étant supposé(s) parfaitement identifiable(s) pour toute unité intermédiaire, on a donc :

$$
\lambda \cdot i+\varphi \cdot i=1 \quad(\forall i=1,2, \ldots, q) .
$$

Cela revient encore à écrire que :

$$
\mathbf{U} \cdot \mathbf{M}_{\mathbf{I}}^{\mathrm{II}}+\mathbf{U} \cdot \mathbf{M}_{\mathbf{F}}^{\mathrm{CI}}=\mathbf{U}
$$

\section{La constitution des matrices de contrôle ultime}

Les raisonnements adoptés pour construire les matrices de possession ultime sont transposables aux matrices de contrôle ultime :

- des agents finaux :

$$
\begin{aligned}
& \mathbf{M}_{\mathbf{P}}^{\mathrm{CU}}=\mathbf{M}_{\mathbf{P}}^{\mathrm{CI}} \cdot\left(\mathrm{I}-\mathbf{M}_{\mathrm{I}}^{\mathrm{CI}}\right)^{-\mathbf{1}} \mathbf{1} \\
& \mathbf{M}_{\mathbf{P}}^{\mathrm{CU}}=\left(\omega h_{i}\right) \in \mathbf{M} p, q(\mathrm{~V})
\end{aligned}
$$

- des unités intermédiaires :

$$
\begin{gathered}
M_{I}^{\mathrm{CU}}=M_{I}^{\mathrm{CI}} \cdot\left(\mathrm{I}-\mathbf{M}_{\mathbf{I}}^{\mathrm{CI}}\right)^{-1} \\
M_{\mathrm{I}}^{\mathrm{CU}}=(\varepsilon i i) \in \mathbf{M} \boldsymbol{q}(\mathrm{V}), 0 \leqslant \varepsilon \cdot i \leqslant 1 .
\end{gathered}
$$

Notons que les agents finaux ont le contrôle ultime de toutes les unités intermédiaires $\left(U \cdot M_{q}^{c U}=U\right.$ ou $\omega \cdot j=1(\forall j=1,2, \ldots, q)$ ).

1. Si $\left(\mathrm{I}-\mathbf{M}_{\mathbf{I}}^{\mathrm{CX}}\right)$ est régulière. La remarque concernant $\left(\mathbf{I}-\mathbf{M}_{\mathbf{I}}^{\mathrm{PI}}\right)$ vaut également ici. 


\section{L'appréhension des groupes}

Un groupe est un ensemble d'unités économiques qui exercent leurs activités sous le contrôle d'une unité centrale. L'identification des têtes de groupe permet donc le repérage des groupes dans le système productif. Ces têtes de groupe figurent en fait (avec les unités indépendantes) parmi les unités intermédiaires contrôlées par les agents finaux.

Le calcul du vecteur $U \cdot M_{F}^{o x}$ de dimension $(1, q)$ permet d'identifier les unités intermédiaires indépendantes ou les têtes de groupe, celles-ci correspondant aux composantes non nulles du vecteur. Ce vecteur composera la diagonale principale d'une matrice carrée d'ordre $q$ où tous les autres coefficients sont nuls $\left(\widehat{U \cdot M_{P}^{G 1}}\right)$. Cette matrice sera dite matrice de contrôle des têtes de groupes $\left(M_{\mathrm{ra}}^{\mathrm{c}}\right)$. On peut dès lors déterminer, selon la démarche précédente, la matrice de contrôle ultime des têtes de groupes, dénommée matrice des groupes $\left(\mathbf{M}_{G}{ }_{G}\right)$

$$
M_{G}^{c}=M_{T a}^{c} \cdot\left(I-M_{I}^{C x}\right)^{-1}
$$

avec

$$
\left.M_{G}^{0}=(\mu i j) \in M q(V), \mu \cdot i=1 \text { o }{ }_{G} i=1,2, \ldots, q\right)^{1}
$$

L'appartenance à un groupe (ou le caractère indépendant) de toute unité intermédiaire $y$ est parfaitement déterminée. Plus précisément, dans la matrice $\mathbf{M}_{G}{ }_{G}$, on lit en ligne les contrôles directs et indirects. Ainsi si sur la $i$ ième ligne :

$-\mu i j=0(\forall j=1,2, \ldots, q)$, alors l'unité $i$ n'est pas tête de groupe. Elle appartient à un groupe que l'on peut identifier en lisant la $i$ ième colonne;

- il existe au moins un $\mu i j \neq 0$, alors :

- si l'élément diagonal est le seul qui soit non nul, l'unité $i$ est indépendante ;

- s'il existe plusieurs $\mu i j \neq 0, i$ est la tête de groupe des unités $j$ à coefficient non nul.

On peut d'ailleurs construire et lire de manière similaire la matrice de possession des groupes $\left(\mathbf{M}_{\mathbf{G}}^{\mathbf{P}}\right)$.

\section{Les indicateurs de décalage possession-contrôle}

Le décalage qui existe entre contrôle et possession, que l'on peut taxer de dualité des rapports de propriété, est à présent aisément mesurable par soustraction des matrices correspondantes.

On obtient ainsi l'indicateur de décalage :

- des agents finaux : $\Delta_{\mathbf{F}}=\mathbf{M}_{\mathbf{F}}^{\mathrm{cJ}}-\mathbf{M}_{\mathbf{P}}^{\mathrm{PJ}}$

1. Se reporter pour démonstration à louvrage précité de F. Morin, p. 38-39. 
avec par construction

$$
\Delta_{\mathbf{p}}=\left(\delta h_{\eta} \in M p, q(W), W=\{x \in Q / x \in[-1,1]\}\right.
$$

- des groupes : $\Delta_{G}={M^{c}}_{G}-M_{G}^{P_{G}}$

$$
\Delta_{a}=\left(\delta^{\circ} i \text { i }\right) \in M q(W) .
$$

Notons d'ailleurs que $U \cdot \Delta_{\mathbf{r}}=\mathrm{U} \cdot \Delta_{\mathrm{G}}=\mathbf{0}_{\mathrm{Rq}}$

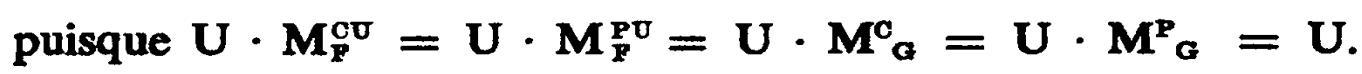

Cela revient à écrire $\delta \cdot j=\delta^{\prime} \cdot j=0(\forall j=1,2, \ldots, q)$.

Chaque coefficient des matrices permet de jauger un décalage possession-contrôle :

- si $\delta h j$ ou $\delta^{\prime} i j<0$, l'unité $h$, respectivement $i$, exerce un pouvoir plus faible que le montant de ses apports dans $j$. Ces * bailleurs de fonds $\gg$ ont un coefficient extrême de -1 ;

- si $\delta h j$ ou $\delta^{\prime} i j=0$, les apports de $h$, respectivement $i$, sont inexistants ou se situent à hauteur du contrôle exercé ;

- si $\delta h j$ ou $\delta^{\prime} i j>0$, l'unité $h$, respectivement $i$, bénéficie dans $j$ d'un pouvoir de contrôle supérieur à la part du capital apporté.

\section{REFORMULATION DE LA REPRÉSENTATION MATRICIELLE DES RAPPORTS DE PROPRIÉTÉ}

La formalisation matricielle des rapports de propriété que nous venons d'exposer n'est pas sans susciter des critiques dès lors que l'on se propose d'en faire un instrument de perception d'ensemble du système de contrôle au sein d'une économie donnée. Aussi serons-nous amené à nous interroger, d'une part, sur la dimension instrumentale de la représentation proposée, d'autre part, sur la pertinence d'une hypothèse implicite centrale : celle de maximisation du contrôle.

Propos sur la dimension instrumentale de la représentation

Les limites internes de la formalisation proposée sont d'abord d'ordre pratique : des problèmes de nature concrète ne sont aucunement abordés dans la théorie du patrimoine. Par ailleurs, l'évaluation du décalage des rapports de propriété est à notre sens assez largement sous-estimée. 


\section{Les difficultés pratiques}

\section{Les «prolongements forcés 》 a l'étranger}

Si l'on entend saisir dans leur ensemble les multiples inter-relations patrimoniales qui sous-tendent le contrôle des unités intermédiaires nationales, il n'est pas possible de restreindre le champ opératoire à l'économie considérée. Il faut bien évidemment prendre en considération les agents étrangers détenteurs de titres de propriété dans les unités économiques nationales. La formalisation repose d'ailleurs sur la connaissance exhaustive de la composition du capital social de toutes les unités économiques intermédiaires. On retrouve cette contrainte dans les matrices. Par exemple, les matrices de possession immédiate des agents intermédiaires $(\alpha i j)$ et finaux $\left(\beta h_{j}\right)$ vérifient la contrainte $: \alpha \cdot j+\beta \cdot j=1$ $(\forall j=1,2, \ldots, q)$. La prise en compte de $m(m<p)$ agents étrangers dans les matrices des agents finaux ne pose d'ailleurs pas de problème particulier. Il n'en est pas de même des unités économiques intermédiaires détentrices d'homologues nationaux. Ces unités étrangères apparaissent alors en lignes des matrices des unités intermédiaires et donc, par contrainte de construction, en colonnes. Mais ces colonnes doivent également avoir pour total l'unité, ce qui conduit à identifier les détenteurs (finaux ou intermédiaires, français ou étrangers) de ces nouvelles unités. Les unités finales étrangères devront alors figurer dans les matrices des agents finaux, les unités intermédiaires étrangères en colonnes des matrices des agents intermédiaires...

Aussi est-il nécessaire de remonter toute la chaîne des possessions des détenteurs étrangers implantés dans l'économie considérée. Autant dire que, par-delà la lourdeur des opérations, on se trouve confronté ici, plus encore qu'au plan national, à d'importantes difficultés d'accès à l'information.

\section{L'éparpillement du capital social de certaines unités intermédiaires}

Il est proposé, dans la théorie du patrimoine, de construire les matrices de contrôle immédiat par simple lecture des matrices de possession immédiate. Implicitement, la connaissance des seuls taux de participation est donc supposée fournir des informations suffisantes pour identifier sans équivoque possible les agents de contrôle de toute unité économique intermédiaire. Or, dans de nombreuses unités économiques intermédiaires, on assiste à une dissémination telle du capital social qu'aucun agent de contrôle n'apparaît clairement. La seule connaissance des taux de participation dans ces unités ne permettant pas d'écrire les matrices de contrôle, il faut donc recourir à d'autres éléments d'appréciation du pouvoir réel.

Il s'agit ici de percevoir des stratégies d'alliances qui naissent au niveau des unités intermédiaires et débouchent sur un contrôle partagé. L'examen des matrices de possession ultime des agents intermédiaires 
et finaux peut parfois fournir des indications éclairantes sur la nature réelle du pouvoir dans ces unités intermédiaires en permettant d'appréhender l'ensemble des participations directes et indirectes de chaque agent dans l'unité considérée. On pourra ainsi octroyer le contrôle immédiat à l'une des unités possédées par l'agent y étant le mieux implanté. Mais le repérage des alliances objectives contractées au sein des unités intermédiaires peut nécessiter, par ailleurs, l'obtention d'éléments d'information supplémentaires sur les comportements stratégiques des agents au sein des conseils d'administration. Il est alors possible, le cas échéant, de s'assurer de l'adéquation des grands axes stratégiques de l'unité considérée avec ceux des unités ou des agents (et des unités qu'ils dirigent) censés en avoir le contrôle ${ }^{1}$.

L'éparpillement du capital social ne constitue cependant pas la seule difficulté pour construire les matrices de contrôle. La lecture des matrices de possession immédiate conduit également à des erreurs d'appréciation sur le contrôle effectif de certaines unités économiques et de ce fait à une sous-estimation du décalage des rapports de propriété.

\section{La sous-évaluation du décalage des rapports de propriété}

La seule prise en compte des rapports d'appropriation juridique ne permet pas de rendre compte dans toute son ampleur du décalage possession-contrôle. Le contrôle d'unités économiques peut se trouver intériorisé (on parle d'autocontrôle) ou avoir une origine externe. Dans ces conditions, l'appréhension du décalage effectif possession-contrôle amène à compléter l'analyse en intégrant les dimensions non patrimoniales de l'appropriation économique.

\section{L'autocontrôle}

Le développement de l'autocontrôle n'a pas échappé à F. Morin mais ne fait l'objet que d'un examen à part, en dehors du schéma proposé sur le décalage possession-contrôle. Pourtant, il s'agit d'un mode d'appropriation économique qui, n'étant pas fondé sur la détention de titres de propriété, accentue plus encore ce décalage. Il marque en effet l'émergence, parmi les détenteurs finaux disposant d'un pouvoir économique important, d'agents sans patrimoine mobilier correspondant. Rien n'empêche cependant de prendre en considération dans l'analyse cette dimension supplémentaire.

Il suffit d'identifier les bénéficiaires de l'autocontrôle et de les faire figurer dans les matrices. Dans la matrice de contrôle immédiat, ils se

1. Notons à ce propos que l'on peut s'émanciper de l'hypothèse implicite qui fonde chez F. Morin la construction des matrices de contrôle immédiat, selon laquelle le contrôle des unités intermédiaires est partagé égalitairement. L'appréhension des rapports de force et des alliances contractées entre codétenteurs des unités intermédiaires pourrait déboucher sur la construction de taux de contrôle sur chaque société qui, le cas échéant, diffèrent d'un agent de contrôle à un autre, mais dont la somme reste égale à lunité. 
verront alors affecter des coefficients non nuls pour les unités intermédiaires qu'ils contrôlent malgré la faiblesse (voire la nullité) des coefficients correspondants dans la matrice de possession immédiate. En conséquence, les agents (finaux ou intermédiaires) détenteurs du capital des unités intermédiaires considérées se voient, conformément à la réalité, privés d'un contrôle qu'on leur avait octroyé par simple prise en compte des rapports de possession.

On va donc transformer les matrices $M_{I}^{\text {or }}$ et $M_{\mathrm{F}}^{\text {or }}$ en $\mathbf{M}_{\mathrm{I}}^{\mathrm{OI}}$ et $\mathbf{M}_{\mathrm{P}}^{\mathrm{OI}}$ de

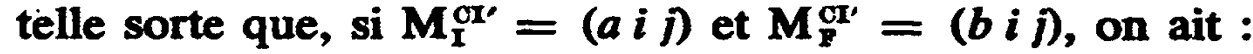

$$
a \cdot i+b \cdot i=1 \quad(\forall i=1,2, \ldots, q)
$$

avec $M_{\mathrm{I}}^{\mathrm{Cr}} \in M_{q}(\mathrm{~V})$ et $M_{\mathrm{P}}^{\mathrm{Cr}} \in \mathrm{M}^{\prime}, q(\mathrm{~V}), p^{\prime}$ étant le nombre d'agents finaux après intégration (si ce n'est le cas) de ceux bénéficiant de l'autocontrôle $\left(p^{\prime} \geqslant p\right)$.

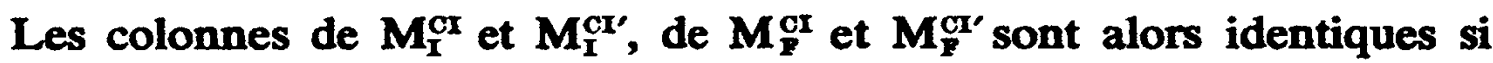
les unités intermédiaires correspondantes ne sont pas autocontrôlées ; dans le cas contraire, une réallocation des coefficients est opérée sur les colonnes concernées.

A partir des matrices $M_{I}^{\text {cI' }}$ et $M_{\mathbf{P}}^{\text {cl' }}$, on peut reconstruire la matrice de contrôle ultime des agents finaux $\left(M_{F}^{\mathrm{CU}^{\prime}}\right)$ :

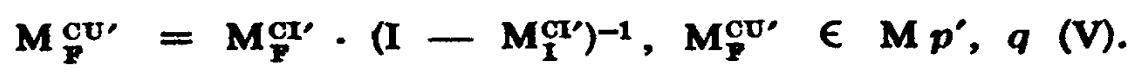

On va déterminer ici l'indicateur de décalage de contrôle de la propriété autocontrôlée :

$$
\Delta \text { auto }=\mathbf{M}_{\mathbf{p}}^{\mathrm{qu}}-\left(\mathbf{M}_{\mathbf{p}}^{\mathrm{cu}}\right)_{t}
$$

où $\left(M_{\mathbf{p}}^{\mathrm{cv}}\right)_{t}$ est la matrice de $M p^{\prime}, q(V)$ telle que :

$$
\left(M_{P}^{G O}\right)_{t}=\left(M_{P}^{C I}\right)_{t} \cdot\left(I-M_{I}^{C I}\right)^{-1}
$$

Nous appelons $(M,)_{t}$ la matrice de dimension $\left(p^{\prime}, q\right)$ qui diffère de $\mathbf{M}_{\mathrm{F}}^{\mathrm{CI}}$ par introduction (le cas échéant) de lignes nulles pour les bénéficiaires de l'autocontrôle (lignes positionnées dans $M_{Y}^{\mathrm{Cr}^{\prime}}$ ).

On voit donc que $\Delta$ auto $\in M^{\prime}, q(W)$ puisqu'elle est construite par soustraction de matrices de dimension $\left(p^{\prime}, q\right)$ à coefficients rationnels compris entre 0 et 1 .

Posons $\Delta$ auto $=(c k j)$. Alors :

- Si $c k j<0$, l'agent $k$ se voit privé du contrôle exclusif (cas où $c k j=-1)$ ou conjoint de l'unité intermédiaire $j$ que devrait « théoriquement , lui conférer son patrimoine en l'absence d'autocontrôle de l'unité $j$ ou d'une unité qui en a le contrôle.

- Si $c k j>0$, l'agent $k$ participe seul $(c k j=1)$ ou conjointement au contrôle de l'unité $j$ par autocontrôle direct ou indirect. 
- Si $c k j=0$, soit l'agent $k$ ne subit pas, du fait de l'autocontrôle de $j$, d'érosion de son contrôle potentiel ; soit la société $j$ n'est pas autocontrôlée (de manière directe ou indirecte) et alors la $j$ ième colonne de $\Delta$ auto n'est composée que de termes nuls.

On peut, par ailleurs, redéfinir :

- la matrice de contrôle ultime des unités intermédiaires :

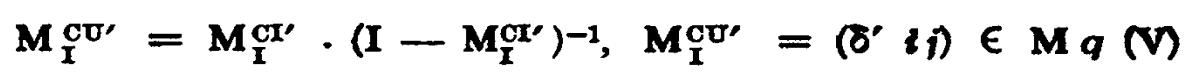

- la matrice des têtes de groupes :

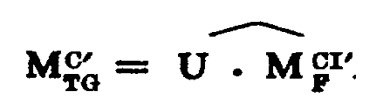

$\mathrm{M}_{\mathrm{T} Q}^{\mathrm{C}}$ étant une matrice diagonale de dimension $(q, q)$

- la matrice de contrôle des groupes :

$$
\begin{aligned}
& \mathbf{M}_{\mathbf{G}}^{\mathbf{C}^{\prime}}=\mathbf{M}_{\mathbf{T Q}}^{\mathbf{C}^{\prime}} \cdot\left(\mathbf{I}-\mathbf{M}_{\mathbf{I}}^{\mathbf{C r}^{\prime}}\right)^{-\mathbf{I}} \\
& M_{\mathbf{a}}^{C^{\prime}}=\left(\mu^{\prime} i j\right) \in M q(V) \text { avec } \mu^{\prime} \cdot i=1 \quad(\forall j=1,2, \ldots, q)
\end{aligned}
$$

\section{La domination externe non patrimoniale d'unités intermédiaires}

L'appropriation économique traditionnelle se trouve également partiellement remise en cause par l'existence de rapports de domination par le crédit ou par le marché (notamment par sous-traitance). Certaines unités économiques (des PME) font ainsi partie de ce que l'on appelle la mouvance des groupes financiers et industriels. Ce type d'appropriation économique - non fondé sur la détention de titres de propriété - est donc également la source d'un décalage propriété-contrôle. Pour appréhender ce phénomène, il va donc d'abord s'agir d'identifier les unités dépendantes au point qu'un réel divorce s'instaure entre appropriations juridique et économique. Cette tâche est réellement ardue. Pour guider l'analyse, on peut cependant s'appuyer sur :

- une matrice de créances-dettes immédiates des unités intermédiaires :

$$
\mathbf{M}_{\mathbf{I}}^{\mathrm{CD}}=(d i i) \in \mathbf{M} q(\mathrm{~V})
$$

où $d i j$ représente la part de l'endettement contractée par l'unité $j$ auprès de l'unité i;

on a :

$$
0 \leqslant d . i \leqslant 1 \quad(\forall i=1,2, \ldots, q)
$$

- une matrice de part de marché immédiate des unités intermédiaires

$$
\mathbf{M}_{\mathbf{I}}^{\mathbf{P}}=\langle\boldsymbol{e} \boldsymbol{i} i) \in \mathbf{M} q(\mathrm{~V})
$$


où $e i j$ est la part des ventes de $j$ à $i$;

avec

$$
0 \leqslant e \cdot i \leqslant 1 \quad(\forall i=1,2, \ldots, q) .
$$

$d \cdot j$ et $e \cdot j$ ne sont pas forcément égaux à l'unité, car :

- l'endettement ou les ventes auprès d'unités étrangères peuvent échapper au recensement ;

- l'endettement et les ventes auprès d'unités finales ne sont pas pris en considération.

On peut alors construire les matrices d'endettement et de vente des unités intermédiaires à l'égard des groupes :

$$
\mathbf{M}_{\mathbf{G}}^{\mathrm{CD}}=\mathbf{M}_{\mathbf{a}}^{\mathbf{C}} \cdot \mathbf{M}_{\mathbf{I}}^{\mathbf{C D}} \text { et } \mathbf{M}_{\mathbf{G}}^{\mathbf{P M}}=\mathbf{M}_{\mathbf{G}}^{\mathbf{C}^{\prime}} \cdot \mathbf{M}_{\mathbf{I}}^{\mathrm{PM}}
$$

où $M_{a}^{c}$ est la matrice de contrôle ultime des groupes (compte tenu de la propriété autocontrôlée).

Posons $M_{\mathrm{a}}^{\mathrm{CD}}=(f i j)$ et $\mathbf{M}_{\mathrm{a}}^{\mathrm{PM}}=(g i j)$; alors $0 \leqslant f \cdot j \leqslant 1$ et $0 \leqslant g \cdot j \leqslant 1(\forall j=1,2, \ldots, q)$, puisque $M_{G}^{\mathrm{CD}}$ et $M_{\mathrm{G}}^{\mathrm{PM}}$ sont issues du produit de matrices à coefficients compris entre 0 et 1 et où la somme de chaque colonne est inférieure ou égale à 1.

Par construction, $M_{G}^{\mathrm{CD}}$ et $\mathbf{M}_{\mathrm{G}}^{\mathrm{PM}}$ appartiennent à $M q(\mathrm{~V}) ; f i j$ et $g$ if représentent alors la part de l'endettement, respectivement des ventes, que l'unité $j$ réalise (le cas échéant) auprès du groupe ou de l'unité indépendante $i$.

L'adoption de seuils critiques va permettre d'appréhender le contrôle exercé par le groupe ou l'unité indépendante $i$ sur l'unité $j^{1}$. Nous considérerons que $i$ s'arroge une part au moins de contrôle sur $j$ si :

$$
\begin{aligned}
{\left[f_{i} i>\right.} & f 1] \vee\left[g_{i j}>g \text { l] } \vee[(f i f>f 2) \wedge(g i j>g \text { 2)] }\right. \\
& \quad f 1, f 2, g 1, g 2 \in V ; f 2<f 1 \text { et } g 2<g 1^{2} .
\end{aligned}
$$

Si nous appelons $t^{c} i j$ le taux de contrôle de $i$ sur $\mathrm{j}\left(0 \leqslant t^{c} i j \leqslant 1\right)$, on a ici $t^{c} i j \neq 0$.

$t^{c} i j$ sera par ailleurs égal à 1 si :

$$
[f i f \geqslant f 3] \vee[g i j \geqslant g \text { 3] } \vee[(f i j \geqslant f 4) \wedge(g i j \geqslant g 4)] 3
$$

$f 3, f 4, g 3, g 4 \in V ; f 1<f 3, f 4<f 3, g 1<g 3, g 4<g 32$.

1. On pourra cependant retenir préalablement un seuil minimal d'endettement de l'unité considérée (ratio d'autonomie financière : dettes de toutes natures sur capitaux propres) pour engager la procédure des transferts de contrôle envisagée ici.

2. Ces seuils (ou taux) pourraient fort bien être déterminés par enquêtes.

3. On pourrait considérer qu'au delà d'un certain taux d'endettement global d'une unité les banques créditrices s'entendent pour s'en partager le contrôle. 
Adoptons l'hypothèse de progressivité linéaire du taux de contrôle afin de déterminer $t^{c} i j$ pour les cas de contrôle partiel obtenu :

- par le seul endettement $(f 1<f i j<f 3)$

$$
t_{i j} i=\frac{f i i-f i}{f 3-f 1}
$$

- par les seules ventes $(g 1<g i j<g 3)$

$$
t^{c} i j=\frac{g i j-g 1}{g^{3}-g 1}
$$

- par l'action conjointe de l'endettement et des ventes

$$
\begin{gathered}
(f 2<f i j<f 4) \wedge(g 2<g i j<g 4) \\
t^{c} i i=\frac{\alpha \cdot(f i i-f 2)+\beta \cdot(g i j-g 2)}{\alpha \cdot(f 4-f 2)+\beta \cdot(g 4-g 2)}
\end{gathered}
$$

avec $\alpha+\beta=1$, $\alpha$ et $\beta$ représentant les pondérations respectives de l'endettement et des ventes dans l'octroi du contrôle ${ }^{1}$.

Si la $j$ ième unité est contrôlée de manière externe par l'unité $i$, on remplacera alors par $t^{c} i j$ le coefficient $\varepsilon^{\prime} i j$ de la matrice de contrôle ultime des unités intermédiaires $\left(M_{\mathfrak{I}}^{\mathrm{CJ}}\right)$. En conséquence, les précédents contrôles d'origine patrimoniale sur l'unité $j$ seront réduits, qu'ils émanent d'unités intermédiaires ou finales. Pour ce faire, nous remplacerons, sur la $j$ ième colonne de $M_{\mathrm{f}}^{\text {cU' }}$ et de $M_{*}^{\mathcal{C U}^{\prime}}$, les termes $\varepsilon^{\prime} f j(f \neq i)$ et $\omega^{\prime} l j$ par, respectivement, $\varepsilon^{\prime} f j \cdot\left(1-t^{c} i j\right)$ et $\omega^{\prime} l j \cdot\left(1-t^{c} i j\right)^{2}$. On obtient ainsi de nouvelles matrices de contrôle ultime des agents intermédiaires et finaux notées :

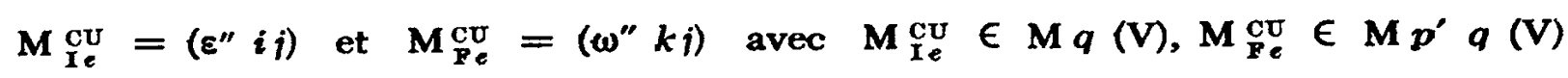
et $\omega^{\prime \prime} \cdot j=1(\forall j=1,2, \ldots, q)$.

Après avoir déterminé $M_{\mathrm{Ie}}^{\mathrm{Cl}}$ et $\mathrm{M}_{\mathrm{Pe}}^{\mathrm{Cr}}{ }^{3}$, on va reconstruire la matrice de contrôle des groupes : $\mathbf{M}_{\mathrm{G} e}^{\mathrm{C}}=\left(\widetilde{U} \cdot \widehat{M}_{\mathrm{P}_{e}}^{\mathrm{CI}}\right) \cdot\left(\mathrm{I}-\mathbf{M}_{\mathrm{I} \ell}^{\mathrm{CI}}\right)^{-1}$.

1. Bien entendu, des perfectionnements sont envisageables : on pourrait attribuer à l'endettement de court terme un poids tout particulier dans la transmission de contrôle par endettement et définir des seuils de dépendance à l'égard d'un fournisseur conduisant à la perte de contrôle.

2. Nous avons raisonné jusqu'à présent en supposant que $i$ n'exerce pas de contrôle financier (partagé) sur $j$. Si tel est le cas, on peut penser que la coexistence de sources différentes de pouvoir va permettre à $i$ de s'arroger un contrôle exclusif sur $i$.

3. On peut déterminer $M_{I}^{\mathrm{cr}}$ à partir de $M_{I}^{\mathrm{cU} e}$. En effet :

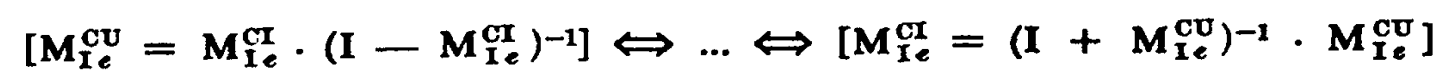

$M_{P e}^{\text {Cr }}$ s'obtient par suite de la manière suivante :

$$
\mathbf{M}_{\mathbf{P}}^{\mathrm{Cr}}=\mathbf{M}_{\mathbf{P}}^{\mathrm{CU}} \cdot\left(\mathbf{I}-\mathbf{M}_{\mathbf{I}}^{\mathrm{CX}}\right)
$$


Alors $M_{a_{e}}^{\mathrm{o}}=\left(\mu^{\prime \prime} i j\right) \in M_{q}(\mathrm{~V})$ et $\mu^{\prime \prime} \cdot j=1(\forall j=1,2, \ldots, q)$.

On peut des lors percevoir l'incidence de la domination externe des unités économiques en calculant :

$$
\Delta \text { ext }=M_{p e}^{\mathrm{CU}}-M_{p}^{\mathrm{CU}^{\prime}} \quad \Delta \text { ext } \in M_{p^{\prime}}, q(W) .
$$

Posons $\Delta$ ext $=(v k j)$.

Les coefficients $v \boldsymbol{k} j$ strictement positifs permettent d'appréhender les agents finaux $(k)$ benéficiant d'un contrôle externe dans certaines unités $(j)$; les coefficients strictement négatifs, les agents finaux se voyant privés d'un contrôle malgré leurs titres de propriété.

Enfin, on va pouvoir déterminer l'indicateur de décalage élargi possession-contrôle :

- des agents finaux : $\Delta_{\mathbf{r}}^{e}=\mathbf{M}_{\mathbf{r}}^{\mathrm{CU}}-\left(\mathbf{M}_{\mathbf{P}}^{\mathrm{PU}}\right)_{\boldsymbol{t}}$

$\left(M_{P}^{P U}\right)_{t}$ s'obtient par une transformation de $M_{P}^{P 0}$ qui en fait une matrice à $p^{\prime}$ lignes, $c^{\prime}$ est-à-dire en y insérant des lignes nulles pour les agents d'autocontrôle identifiés précédemment.

- des groupes : $\Delta_{\mathrm{G}}^{\mathrm{e}}=\mathbf{M}_{\mathrm{G} e}^{\mathrm{C}}-\mathbf{M}_{\mathrm{G}}^{\mathrm{P}_{\mathrm{G}}}$

où $\Delta^{e} \mathbf{r}=\left(\delta^{e} k j\right) \in M p^{\prime}, q(W)$

et $\Delta_{\mathrm{a}}^{e}=\left(\delta^{\prime e} i j\right) \in M q(\mathrm{~W})$

$\operatorname{avec} \delta^{e} \cdot j=\delta^{\prime e} \cdot j=0(\forall j=1,2, \ldots, q)$,

l'interprétation des matrices $\Delta_{F}$ et $\Delta_{G}$ valant pour $\Delta^{e}$ et $\Delta^{e}{ }_{G}$.

\section{La renonciation à l'hypothèse d'uniformité comportementale des agents en matière de contróle}

La démarche retenue plus haut repose implicitement sur l'hypothèse de maximisation (ou encore sur une perception maximaliste) du contrôle exercé par les agents intermédiaires ou finaux en position de faire valoir leurs prérogatives. Le passage des matrices de possession aux matrices de contrôle immédiat est ainsi fondé sur l'idée que l'agent considéré s'assure le contrôle de toute unité intermédiaire dès lors que ses titres de propriété sont quantitativement suffisants, sauf, comme nous l'avons montré mais qui ne modifie pas fondamentalement la méthode, en cas de surdétermination extra-patrimoniale. Or les comportements des agents économiques en matière de contrôle ne peuvent être transcrits aussi synthétiquement. Une dissociation public-privé s'impose à ce niveau, l'Etat n'adhérant pas totalement à la logique de maximisation du contrôle. Car la volonté de l'Etat de ne pas par trop empiéter sur le domaine privé ou encore la disponibilité des capitaux publics pour des missions de dynamisation du secteur privé, amène parfois des unités publiques à renoncer à s'assurer le contrôle d'unités intermédiaires, alors qu'elles disposent 
d'un potentiel (titres de participation ou autres sources de domination) suffisant. C'est dire qu'existent conjointement dans la sphère étatique des comportements diamétralement opposés en matière de contrôle. Le problème consiste alors à repérer les unités intermédiaires sur lesquelles s'exerce cette spécificité comportementale publique afin de juger de l'incidence globale du renoncement public sur le contrôle effectif d'unités productives.

Il faut donc procéder à un traitement particulier des participations directes et indirectes censées conférer à l'Etat le contrôle final d'unités intermédiaires. Une analyse rétrospective des comportements publics en matière de contrôle montre que l'on peut, a priori, mettre à part les participations des organismes parapublics.

Ces organismes parapublics ne s'ingèrent que très rarement dans la gestion des unités intermédiaires où ils détiennent une participation et constituent en fait des \& associés publics dormants ». Leurs participations ne se trouvant pas, sauf exception, à l'origine d'une extention de la sphère de contrôle de l'Etat, elles ne doivent pas être prises en considération pour construire les matrices de contrôle immédiat. Cette première source de décalage spécifique à la propriété publique est aisément mesurable. On va pour cela élaborer de nouvelles matrices de contrôle ultime à partir d'une matrice de contrôle immédiat des unités intermédiaires $\left(\mathbf{M}_{1 o}^{\mathrm{CI}}\right)$ tirée de $\mathbf{M}_{\mathrm{I}}^{\mathrm{CI}}$ en ne prenant pas en compte les détentions des organismes parapublics ${ }^{1}$. Puis on leur retranchera les matrices de contrôle ultime telles qu'elles ressortaient de l'approche agrégative antérieure. Cela nous permettra d'appréhender l'érosion du contrôle public qui nâ̂t du comportement spécifique des organismes parapublics au niveau :

— des unités intermédiaires :

$$
\Delta_{\mathbf{I} o}^{\mathrm{C}}=\mathbf{M}_{\mathbf{1} 0}^{\mathrm{CU}}-\mathbf{M}_{\mathbf{I}}^{\mathrm{CU}}
$$

où $\mathbf{M}_{\mathrm{I} o}^{\mathrm{cu}}=\mathbf{M}_{\mathrm{I} o}^{\mathrm{cr}} \cdot\left(\mathrm{I}-\mathbf{M}_{\mathrm{I} o}^{\mathrm{CI}}\right)^{-1} ; \Delta_{\mathrm{I} o}^{\mathrm{C}} \in \mathbf{M}_{q} q(\mathrm{~W})$

— des unités finales :

$$
\Delta_{\mathbf{F}_{0}}^{\mathbf{c}}=\mathbf{M}_{\mathbf{F}}^{\mathrm{CU}}-\mathbf{M}_{\mathbf{P}_{e}}^{\mathrm{CU}}
$$

où $\mathbf{M}_{\mathbf{F}_{o}}^{\mathrm{CU}}=\mathbf{M}_{\mathrm{F}_{o}}^{\mathrm{CI}} \cdot\left(\mathrm{I}-\mathbf{M}_{\mathrm{I} o}^{\mathrm{CI}}\right)^{-1} ; \Delta_{\mathbf{p}_{o}}^{\mathrm{C}} \in \mathbf{M}_{p^{\prime}}^{\prime}, q(\mathrm{~W})$

- des groupes :

$$
\Delta_{G o}^{\mathbf{C}}=\mathbf{M}_{G o}^{\mathbf{C}}-\mathbf{D}_{G e}^{\mathbf{c}}
$$

1. La matrice de contrôle immédiat des agents finaux pourra également se trouver modifiée, ces derniers étant des bénéficiaires potentiels du comportement spécifique des organismes parapublics. On construira ainsi la matrice $M_{\text {Po a }}^{\text {à }}$ partir de $M_{\text {Pe }}^{\text {OI }}$.

N.B. : L'érosion de contrôle concerne, non seulement les organismes parapublics, mais également les sociétés publiques qui ne bénéficient pas de l'appoint de voix des organismes parapublics. 
où $\mathbf{M}_{\mathrm{g} o}^{\mathrm{g}}=\left(\mathrm{U} \cdot \widehat{\mathrm{M}_{\mathrm{P}}^{\mathrm{Gre}}}\right) \cdot\left(\mathrm{I}-\mathrm{M}_{\mathrm{I} o}^{\mathrm{OI}}\right)^{-1} ; \Delta_{\mathrm{a}_{0}}^{\mathrm{g}} \in \mathrm{M}_{q}(\mathrm{~W})$.

Posons :

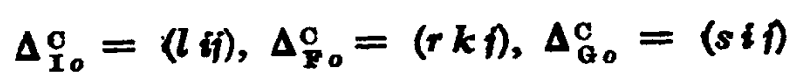

On peut alors dégager précisément l'incidence du comportement particulier des organismes parapublics :

- Si $l i j$ ou $r k j<0$, l'unité $i$ ou l'agent final $k$ (l'Etat) subit dans l'unité $j$ une érosion partielle ou totale (cas où $l i j=-1, r k j=-1$ ) de son pouvoir de contrôle.

- Si $l i j$ ou $r k j=0$, l'unité $i$ ou l'agent final $k$ n'est aucunement affecté dans l'unité $j$ considérée soit parce qu'il n'y existe pas de participation d'organismes parapublics, soit parce que cette ou ces participation(s) ne modifie(nt) pas les situations acquises de contrôle.

- Si $l i j$ ou $r k j>0$, l'unité $i$ ou l'agent final $k$ accroît son pouvoir de contrôle sur l'unité $j$.

Plus encore, on va pouvoir mesurer pour chaque agent son décalage du contrôle en construisant les vecteurs suivants :

$$
D_{\mathbf{I}_{0}}=\Delta_{\mathbf{I}_{0}}^{\mathrm{C}} \cdot \mathbf{U}^{\prime} \text { et } \mathbf{D}_{\mathrm{Fo}_{0}}=\Delta_{\mathbf{p}_{0}}^{\mathbf{C}} \cdot \mathbf{U}^{\prime}
$$

$U^{\prime}$ étant le vecteur colonne de dimension $(q, 1)$ ayant toutes ses composantes égales à l'unité :

$$
D_{\mathrm{x}_{0}} \in M q, 1(\mathrm{~W}) \text { et } \mathrm{D}_{\mathrm{Yo}} \in M p^{\prime}, 1(\mathrm{~W})
$$

Aussi obtient-on $l i \cdot(\forall i=1,2, \ldots, q)$ et $r k \cdot\left(\forall \mathrm{k}=1,2, \ldots, p^{\prime}\right)$ qui permettent de dégager l'ampleur du décalage de contrôle obtenu par l'unité $i$ ou l'agent $k$.

Ces grandeurs peuvent être comparées à l'ampleur du contrôle antérieur des unités ou agents considérés dès lors que celui-ci existait. Cela revient à calculer, si possible (le dénominateur devant être non nul), les valeurs suivantes :

$$
\begin{array}{r}
\left(l i \cdot / \varepsilon^{\prime \prime} i \cdot\right) \text { ou }\left(l i \cdot /\left(\varepsilon^{\prime \prime} i+l i \cdot\right)\right)\left(\forall i=1,2, \ldots, q / \varepsilon^{\prime \prime} i \cdot \neq 0\right. \\
\text { ou } \left.\left(\varepsilon^{\prime \prime} i \cdot+l i \cdot\right) \neq 0\right) \\
\left(r k \cdot / \omega^{\prime \prime} k \cdot\right) \text { ou }\left(r k \cdot /\left(\omega^{\prime \prime} k \cdot+r k \cdot\right)\right)\left(\forall k=1,2, \ldots, p^{\prime} / \omega^{\prime \prime} h \cdot \neq 0\right. \\
\text { ou } \left.\left(\omega^{\prime \prime} k \cdot+r k\right) \neq 0\right)
\end{array}
$$

On peut parfaitement réaffecter les deux premiers groupes de valeurs dans des vecteurs colonne de dimension $(q, 1)$ appelés $V_{1}$ et $V_{1}^{\prime}$; les deux derniers dans des vecteurs de dimension $\left(p^{\prime}, 1\right)$ appelés $V_{2}$ et $V^{\prime}$, où les unités conserveront leurs indices initiaux (celles ne disposant pas de pouvoir de contrôle ne se voyant pas apposer de valeur). 
Par ailleurs, la matrice $\Delta^{\circ}{ }_{G o}$ permet de repérer les groupes bénéficiaires des transferts de contrôle qui naissent des comportements spécifiques des organismes parapublics. Ainsi un $s i j$ positif traduit une prise ou une extension du contrôle du groupe ayant à sa tête $i$ sur l'unité intermédiaire $j$. Inversement, un coefficient $s i j$ négatif traduit une perte de contrôle du groupe (public) $i$ sur l'unité $j$.

Cependant, la renonciation publique ne concerne pas les seuls organismes parapublics : le phénomène touche également, mais de manière ponctuelle, d'autres unités du secteur public. Une fois encore, il s'agit d'un redéploiement du contrôle du public vers le privé qui échappe à une formalisation des rapports de propriété économique fondée sur la simple lecture de matrice de possession. Reste qu'appréhender l'incidence de ces stratégies de renonciation au contrôle, notamment sur le décalage des rapports de propriété, est ici plus délicat. Car ces opérations présentent un caractère occulte évident (relevant souvent d'injonctions souterraines de l'Etat) d'où les difficultés d'identification des unités soumises au transfert. Ces transferts s'opérant du public vers le privé, on peut s'appuyer sur la construction d'une matrice étatique pour faciliter l'analyse.

Mais, au préalable, il faut s'interroger sur le contrôle immédiat de l'Etat, celui-ci pouvant renoncer à exercer son propre pouvoir de contrôle sur des unités intermédiaires. Cela amène à réexaminer la dernière matrice de contrôle immédiat des agents finaux $\left(\mathbf{M}_{\mathbf{F}_{o}}^{\mathrm{CI}}\right)$ pour, le cas échéant, annuler ou réduire sur la ligne Etat les coefficients relatifs aux unités au sein desquelles il y a aussi renonciation des pouvoirs publics, puis réaffecter ces contrôles à d'autres unités intermédiaires ou finales. Nous appellerons $\mathbf{M}_{\mathrm{F}_{e}}^{\mathrm{CI}}$, cette nouvelle matrice.

On a alors :

$$
U \cdot M_{e^{\prime}}^{\mathrm{Cr}}=U \cdot\left(\mathbf{M}_{E}^{\mathrm{CI}}+\mathbf{M}_{\mathbf{N}}^{\mathrm{Cr}}\right)
$$

$\mathbf{M}_{\mathrm{B}}^{\text {OI }}$ étant la matrice de contrôle immédiat de l'Etat, construite à partir de $M_{P_{e^{\prime}}}^{\mathrm{CI}^{\prime}}$ en $\mathrm{y}$ annulant toutes les lignes hormis la ligne Etat et $\mathbf{M}_{\mathrm{NE}}^{\mathrm{CX}}=\mathbf{M}_{\mathrm{P} \ell^{\prime}}^{\mathrm{CI}}-\mathbf{M}_{\mathrm{R}}^{\mathrm{CI}}$

Or $U \cdot\left(M_{E}^{C I}+M_{N E}^{C I}\right)=\left(U \cdot M_{E}^{C I}\right)+\left(U \cdot M_{N E}^{C I}\right)$.

Alors $M_{\mathrm{T}}^{\mathrm{C} t}=\mathrm{U} \cdot \widehat{M}_{\mathrm{F}_{e^{\prime}}}^{\mathrm{CI}}=\left(\mathrm{U} \cdot \widehat{M}_{\mathrm{B}}^{\mathrm{CI}}\right)+\left(\mathrm{U} \cdot \widehat{M}_{\mathrm{ME}}^{\mathrm{CI}}\right)$

$=\mathbf{M}_{\mathrm{E}}^{\mathrm{o}}+\mathrm{M}_{\mathrm{NE}}^{\mathrm{c}}$, matrices diagonales d'ordre $q$ de contrôle des têtes de groupes publics et non publics.

On en déduit que :

$$
\begin{aligned}
& M_{G}^{C t}=\left(M_{E}^{C}+M_{N \mathbb{R}}^{C}\right) \cdot\left(I-M_{I_{e}}^{C t}\right)^{-1} \\
& =\mathbf{M}_{\mathbf{B}}^{\mathrm{C}} \cdot\left(\mathrm{I}-\mathbf{M}_{\mathbf{I} e^{\prime}}^{\mathrm{CI}}\right)^{-1}+\mathbf{M}_{\mathbb{N}}^{\mathrm{C}} \cdot\left(\mathrm{I}-\mathbf{M}_{\mathbf{I} e^{\prime}}^{\mathrm{CI}}\right)^{-1}
\end{aligned}
$$

On vient de construire les matrices de contrôle :

- des groupes publics : $\mathbf{M}_{\mathrm{GB}}^{\mathrm{O}}=\mathbf{M}_{\mathbf{x}}^{\mathrm{O}} \cdot\left(\mathbf{I}-\mathbf{M}_{\mathbf{I}^{\prime}}^{\mathrm{CI}}\right)^{-1}$

- des groupes non publics : $\mathbf{M}_{\mathbf{G} \mathrm{NB}}^{\mathrm{C}}=\mathbf{M}_{\mathrm{NB}}^{\mathrm{C}} \cdot\left(I-\mathbf{M}_{\mathbf{I} \ell^{\prime}}^{\mathrm{CI}}\right)^{-1}$ 
Posons $M_{\mathrm{Gx}}^{\mathrm{g}}=(t i j)$ et $\mathrm{M}_{\mathrm{Gm}}^{\mathrm{o}}=(y i$ i $) \quad \begin{aligned} & (i=1,2, \ldots, q) \\ & (j=1,2, \ldots, q)\end{aligned}$

Alors par construction $t \cdot j+y \cdot j=1 \quad(\forall j=1,2, \ldots, q)$.

II reste à identifier parmi les unités intermédiaires, figurant dans les matrices des groupes publics, celles qui font l'objet sur directives de l'Etat d'un transfert de contrôle vers le secteur privé, soit par renoncement au contrôle de l'actionnaire public en position de domination potentielle, soit du fait de l'absence d'alliances objectives contractées entre divers participants publics en leur sein (hors organismes parapublics). Les unités soumises a transfert figurent bien évidemment dans la matrice $M_{\mathrm{GE}}^{\mathrm{D}}$. On pourra cependant ne soumettre a analyse que les composantes de cette matrice qui sont pénétrées de manière significative par les capitaux privés. Ces derniers s'appuient en effet sur leur participation (minoritaire) dans les unités potentiellement publiques pour s'en assurer le contrôle. L'examen de la matrice de possession immédiate des unités intermédiaires $\left(\mathbf{M}_{\mathbf{I}}^{\mathrm{PI}}\right)$ permet d'identifier les composantes de $\mathbf{M}_{\mathbf{G} \mathrm{E}}^{\mathbf{T}}$ où figurent des participants privés importants et qui sont par là même susceptibles d'être soumises à des transferts. Les unités à faible majorité publique (surtout si cette majorité relève de sources diverses) et qui sont pénétrées par ces composantes privées de grande taille, présentent d'ailleurs de bien plus grandes chances de sortir de fait du secteur public. Une étude plus fine des comportements stratégiques de ces unités intermédiaires mixtes (portant en particulier sur les convergences avec les stratégies des participants privés) permettra alors de confirmer ou d'infirmer l'existence de transferts de contrôle. Les unités se trouvant dans le premier cas de figure devront être transférées partiellement ou totalement de la matrice $\mathbf{M}_{\mathrm{or}}^{\mathrm{c}} \mathrm{à} \mathrm{la} \mathrm{matrice} \mathbf{M}_{\mathrm{GKg}}^{\mathrm{c}}$. Ainsi, si le groupe public $i$ subit une perte de contrôle dans l'unité intermédiaire $\boldsymbol{j}$ au profit d'une unité du groupe (ou de la société indépendante) $f$, on va annuler le coefficient $t i j$ de $M_{G B}^{c}$ après l'avoir ajouté a la composante y $f j$ de $M_{\mathrm{Grr}}^{\mathrm{C}}$.

On obtient de la sorte les nouvelles matrices du groupe Etat

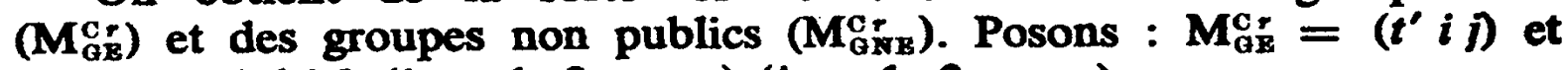
$M_{\mathrm{ors}}^{\mathrm{c} r}=\left(y^{\prime}\right.$ i $)(i=1,2, \ldots, q)(j=1,2, \ldots, q)$ alors $t^{\prime} \cdot j+y^{\prime} \cdot j=1(\forall j=1,2, \ldots, q)$.

Après cette identification des transferts de contrôle qui relèvent de comportements spécifiques des unités publiques (execption faite des organismes parapublics), on peut reconstruire les matrices de contrôle des agents. Il faut d'abord procéder, conformément à ce qui vient d'être établi, à la réallocation des coefficients dans la matrice de contrôle immédiat des unites intermédiaires. On octroiera bien évidemment le contrôle à l'unité privée qui a été identifiée (et qui appartient au groupe $f$ dans le cas précédent) pour en dessaisir une (ou des) unité(s) du groupe public $i$.

Appelons $M_{1 r}^{\text {Gr }}$ cette nouvelle matrice, puis calculons la matrice de contrôle ultime réel des agents intermédiaires : 


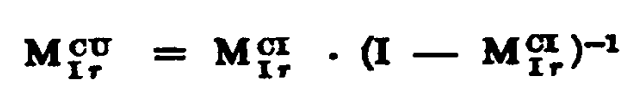

Ecrivons également la matrice de contrôle ultime réel des unités

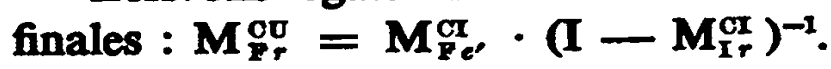

On peut alors calculer le décalage lié à la propriété publique en ce qui concerne :

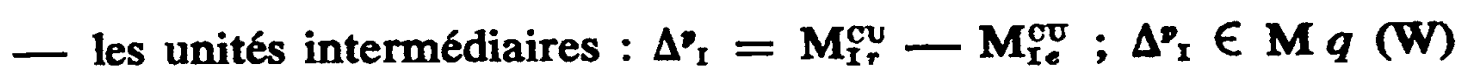

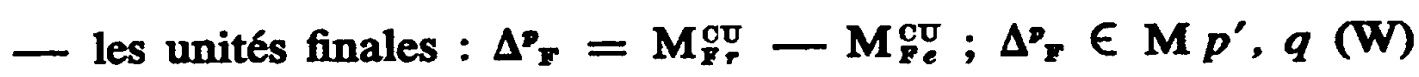

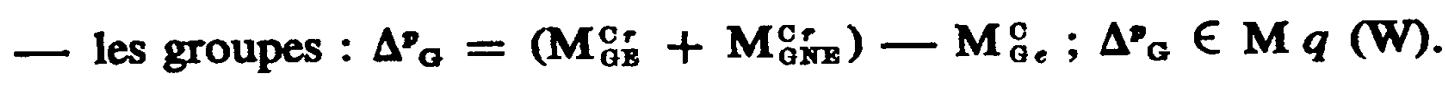

Les remarques que l'on a portées sur les indicateurs du décalage lié au comportement des organismes parapublics valent ici.

On va, par ailleurs, pouvoir déterminer le décalage de contrôle de chaque agent en construisant les vecteurs :

$$
D_{I}^{\infty}=\Delta^{p} \cdot U^{\prime} \text { et } D^{x_{F}}=\Delta \Delta_{F} \cdot U^{\prime}
$$

avec $D_{1}^{p_{1}} \in M q, 1(\mathrm{~W})$ et $D^{p} \in M p^{\prime}, 1(W)$.

Posons : $\Delta^{p_{1}}=\left(l^{\prime} i j\right)$ et $\Delta^{p}{ }_{F}=\left(r^{\prime} k j\right)$ et dégageons, lorsque c'est possible, l'ampleur relative du décalage que subit ou dont bénéficie chaque unité en remplissant, comme précédemment pour $V_{1}, V^{\prime}, V_{2}, V^{\prime}{ }_{2}$, les vecteurs suivants :

$$
\mathrm{W}_{1}:\left(l^{\prime} i \cdot / \varepsilon^{\prime \prime} i \cdot\right) \text { ou } \mathrm{W}_{1}^{\prime}:\left(l^{\prime} i \cdot /\left(\varepsilon^{\prime \prime} i \cdot+l^{\prime} i \cdot\right)\right)
$$

avec $\varepsilon^{\prime \prime} i \cdot$ ou $\left(\varepsilon^{\prime \prime} i \cdot+l^{\prime} i \cdot\right) \neq 0$

$$
\mathrm{W}_{2}:\left(r^{\prime} k \cdot / \omega^{\prime \prime} k \cdot\right) \text { ou } \mathrm{W}_{2}^{\prime}:\left(r^{\prime} k \cdot /\left(\omega^{\prime \prime} k \cdot+r^{\prime} k \cdot\right)\right)
$$

avec $\omega^{\prime \prime} k \cdot$ ou $\left(\omega^{\prime \prime} k \cdot+r^{\prime} i \cdot\right) \neq 0$.

L'appropriation juridique des moyens de production constitue le point d'appui principal de la proriété économique : non seulement elle délimite la situation patrimoniale des agents mais structure également les rapports de domination sur les unités économiques. Aussi peut-on parler, avec F. Morin, de dualité des rapports de propriété. Cette dualité et plus précisément l'inadéquation entre appropriations juridique et économique ont été appréhendées à travers le calcul d'indicateurs de décalage. Cependant, nous avons montré que le décalage des rapports de propriété n'est pas uniquement le reflet d'une structuration du pouvoir s'appuyant sur des différentiels de taux de participation ou même sur l'existence de stratégies d'alliances contractées entre participants dans les unités économiques. Il est en fait bien plus large. Au-delà du décalage relevant de cl'effet de structure de la propriété juridique, des pratiques connexes 
se sont multipliées en matière de contrôle, favorisées d'ailleurs par la fluidification des rapports de possession. Rappelons quelles sont les principales sources de décalage des rapports de propriété et leur formalisation matricielle :

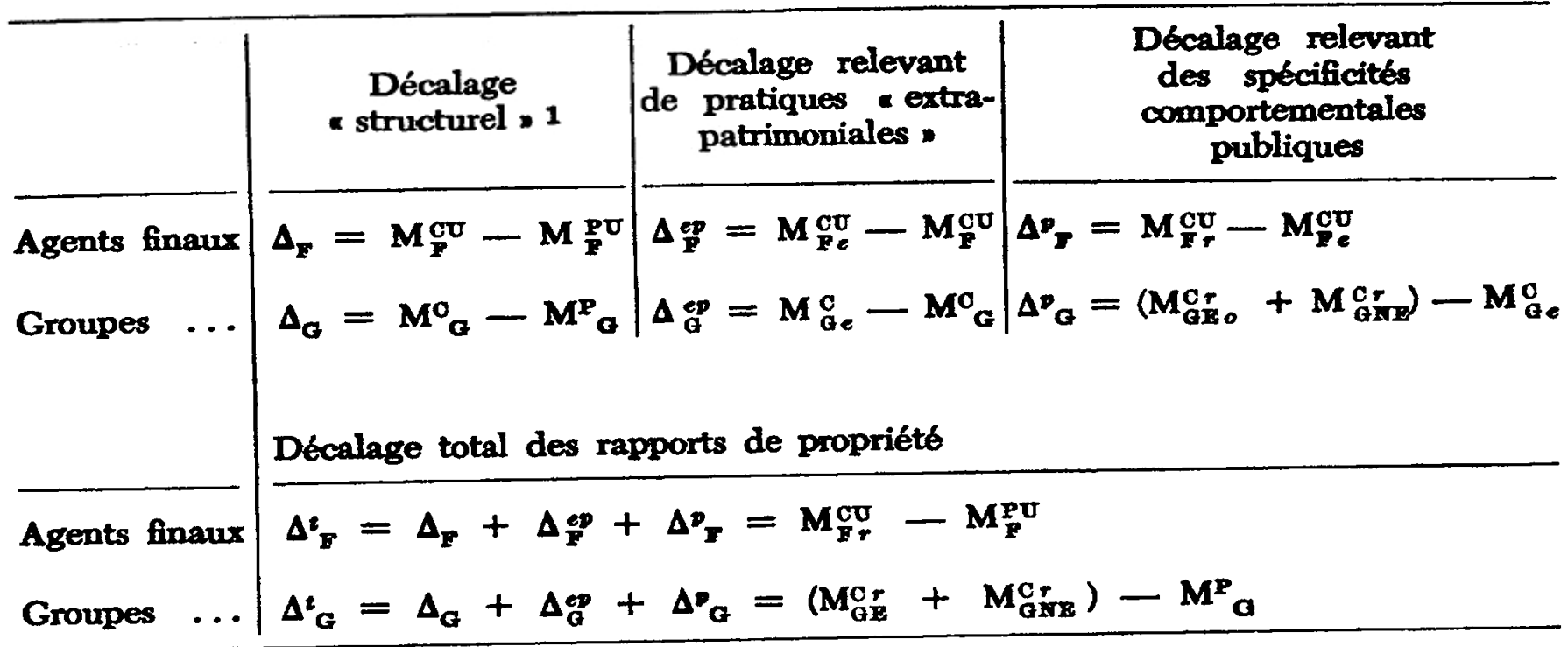

1. Ou = d'effet de structure -.

1. Ou d'effet de structure finaux auront ici pour dimension $\left(p^{\prime}, q\right)$ ce qui revient y inserer $p^{\prime \prime}\left(p^{\prime \prime}=p^{\prime}-p\right)$ IIgnes nulles les rendamx compatibles avec les matrices d'agents fimaux prbeentbes cl-apres.

Reste cependant à se poser une question essentielle : quelles sont les conséquences de ces décalages sur le fonctionnement d'ensemble du système économique? Bien évidemment, les décalages traduisent tous l'existence de déplacements des pouvoirs eu égard à l'appropriation juridique. Ils reflètent ainsi une mobilisation beaucoup plus poussée du capital productif sous l'égide de certains agents au détriment d'autres agents. Peut-on pour autant dire qu'ils marquent l'émergence de pratiques centralisatrices ? En fait, il n'en est ainsi que pour les deux premiers types de décalages ci-dessus énoncés. Car le décalage associé aux comportements spécifiques d'unités publiques constitue au contraire une limitation à la centralisation du capital opérée sous l'égide de l'Etat. De surcroît, on se doit d'opposer le « décalage public » qui relève de comportements spécifiques d'érosion autoprogrammée aux autres types de décalages renvoyant quant à eux à la recherche permanente par les agents d'un élargissement de leur pouvoir (pratiques que l'on peut taxer d'autogènes). Néanmoins, il subsiste un point commun d'importance : ces pratiques sont source de souplesse et de dynamisme au sein des systèmes économiques occidentaux. Les deux premiers facilitent la mobilisation du capital argent ou du capital productif et son affectation à des tâches productives. Quant au décalage public, il traduit non seulement le rejet par l'Etat d'une centralisation publique jugée excessive mais aussi et surtout la recherche d'un plus grand dynamisme du secteur privé par l'entremise d'apports publics. 